\title{
CBIR with Partial Input of Unshaped Images Using Compressed-Pixel Matching Algorithm
}

\author{
Ahilandeswari Thangarajan ${ }^{1 *}$, Vivekanandan Kalimuthu ${ }^{2}$ \\ ${ }^{1}$ Research Scholar, Department of Computer Science and Engineering, Pondicherry Engineering College, Pondicherry, India. \\ ${ }^{2}$ Professor, Department of Computer Science and Engineering, Pondicherry Engineering College, Pondicherry, India. \\ E-mail:kvivek27@gmail.com \\ *Corresponding authorE-mail:meagi123@gmail.com
}

\begin{abstract}
Many works have been done to find out whether given image is in the database using Content Based Image Retrieval (CBIR) techniques. However if the query image is unshaped or noise filled then retrieval of that image in the database is difficult .We propose an approach by which for any shape of input image the databases is searched and the most relevant image is retrieved. Results provides better accuracy than existing one and time elapsed also reduced because of making comparison after compression of both partial image and images from the database. The attainment of the proposed system is assessed using LFW and WANG image sets consisting of 2000 and 9990 images, respectively, and it measured with familiar methods with regard to precision and recall which demonstrates the advantages of the proposed approach.
\end{abstract}

Keywords: Partial input image, content based image retrieval (CBIR), shapes, ROI, compression.

\section{Introduction}

The huge growth of the Internet and digital contents of the Internet creates the greater difficulty to design an effective image retrieval system from wide-ranging database. Content Based Image Retrieval is a technique used to extract analogous desired picture from database of images. There are plentiful methods that based on content for classification and retrieval of an image, are attracted more awareness in recent years. Image retrieval with partial input of shaped or non-shaped image is one of the most important techniques in Image Retrieval system based on Content.

The partial image input retrieval technique is valuable in searching results. In many instances especially in image forensic where only a part of the query image is only available and search needs to be done with only partial image as input. The strength of the proposed method is that the time taken to retrieve the exact image is a reduced when compared to the other CBIR system that takes input as the partial input of an image.

The technique [1] of Object Cropped Image, in which the Local Binary Patterns (LBP) is a non-parametric operation, is described using the native contrast and native spatial structure of an image for texture analyzing and classification. The procedure is tested on only simple image sets.

An asymmetric approach [2] for image retrieval examination, an easy image to image adjacent neighbour categorization method did not lead to competitive accurate categorization. A structure [3] for adaptive image browsing was given based on High-entropy layer distribution (HELD) visualizations. This approach is widely used in interactive browsing and retrieval however limited to work on few query scenarios.

The low-level features of color and texture extraction for CBIR [4], uses color histogram, co-occurrence matrix for retrieving image but are restricted on some low-level features like shape and spatial location. A 2-D multi-index structure together with a new multiIDF formula coupling the SIFT features and the color features at the indexing level, [5] uses Hamming Embedding, soft assignment and multiple assignment techniques. The adoption of postprocessing techniques is necessary to further improve the accuracy. An efficient and effective CBIR system [6] using color, texture and shape-based histograms. However improvement to be made on the effectiveness of the retrieval system.

The new fusion of two types of histograms [7] extracted from color and local directional pattern (LDP)however which has not find the part of information (color information and direction information) which is more importance for retrieval of an image . The graphbased ranking model [8] called Efficient Manifold Ranking (EMR), in this model the anchor graph divided into two parts.one is anchor selection and another one is graph construction which reduces the calculation time, but this may not be a best solution.

\section{Proposed System Architecture}

A new method has been proposed for image retrieval with higher accuracy that work on partial input query image, retrieves query matched image with minimal time. Fig.1 depicts the working principal of the proposed system.

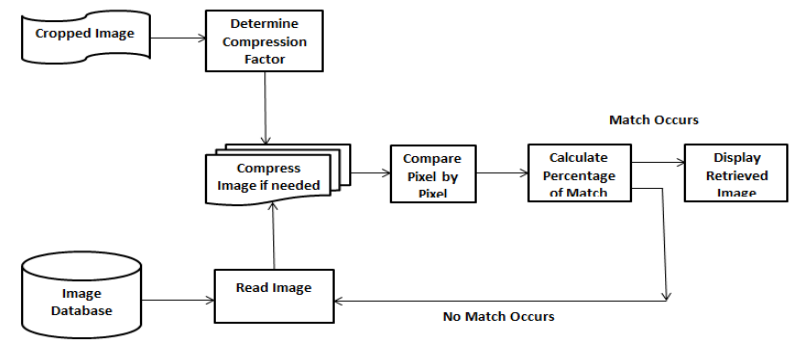

Fig. 1: Structure of the proposed system Copyright $\odot 2018$ Authors. This is an open access article distributed under the Creative Commons Attribution License, which permits unrestricted use, distribution, and reproduction in any medium, provided the original work is properly cited. 
The operation of architecture is explained as follows:

Here the Image database contains organized collection of digital images in jpg format of resolution of $250 \times 250$.

Input: Query color image.

Output: single image retrieved same as that of the query image.

- Querying: To begin a process, an image to be searched is provided which is of unshaped and partial input of the image, the divide aspect vector of the input image is constructed using dimension of a query image.

- Image compression: In this step, the pixel count is downsized for the image to be compared with query image. After compressing both query and target image the similarity measurement is done.

- Resemblance estimation: The method finds the likenesses among the RGB values of searching image and the image taken from the database.

- Retrieval: The retrieval of an image is depends on the matched percentage with query image. In the proposed system the matched percentage is fixed at $17 \%$ based on trial and error method. The system retrieves and displays a single target image as a result.

Advantage of the proposed system is accuracy in image retrieval. Accuracy is the division of number of gathered images like partial input that relates with the user query image. The retrieval time gets reduced because compression is done with reduced search part.

\section{Methodology}

The proposed system is implemented through Matlab 8.3.0.532 (R2014a) [12] which is more suitable for image processing concepts and Java 1.7.0_11-b21 with Oracle Corporation and Java HotSpot (TM) 64-Bit Server VM mixed mode.

Various shapes of the region of interest (ROI) are required to test the proposed method. By using image cropping methods the ROI is taken as sample query images.

By compressing the image the retrieval time of the desired image can be reduced. Then the RGB values of a query image compared pixel-by-pixel with database images. The algorithm is given below:

\section{Algorithm for Compressed Pixel Matching}

Begin

Step 1: Read the query image.

Step 2: Check the dimension of the query image.

Step 3: Calculate Divide Aspect Vector (DAV) based on the query image for compression.

Step 4: Read image from the database.

Step 5: Compress the query image using the DAV which is calculated in step 3 .

Step 6: Apply the same DAV to image that is to be compared from the database.

Step 7: Obtain color image of HSV color space from RGB color space by conversion.

Step 8: Perform pixel-by-pixel comparison.

Step 9: Compute matched percentage among the query and image taken from database.

Step 10: If matched percentage is less than $17 \%$ repeat 4 to step 9 until termination condition met.

Step 11: Display the retrieved image if exists otherwise display the error message.

End

The individual step of this approach is explained below:

\section{ROI}

A region of interest (ROI) is a sub-part taken from an image that the user willing to extract or like to apply different operations on it.So that the operation can be performed on specific region and the remaining regoins are unaffcted.
Image subdivisions may be appropriately measured by means of Mathematica Graphics basics.

\section{Image cropping}

Crafting a new image from a fragment of an original image. Image can be cropped into various shapes like square, rectangle, ellipse, circle and an unshaped image. To crop an image the Image Viewer or Crop Image tool can be used.

\section{Image compression}

Image compression changes the original dimension of an image and can be scaled down to either of the following categories as shown in the Fig. 2(a)-2(c). The divide aspect vector is a number whose value depends on the dimensionality of an original image shown in Table I.

Table I: Divide Aspect Vector

\begin{tabular}{|l|l|l|}
\hline S.no & Image Dimension & Divide Aspect Vector \\
\hline 1 & Less than 500 & 1 \\
\hline 2 & Less than 1000 & 2 \\
\hline 3 & Otherwise & 4 \\
\hline
\end{tabular}

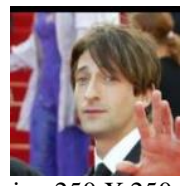

2(a) Original image of dimension 250 X 250 where divide aspect vector $=1$

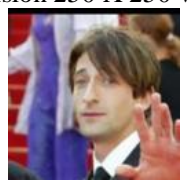

2(b) Image of dimension $125 \times 125$ where divide aspect vector $=2$

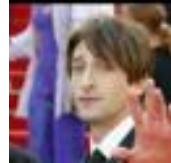

2(c) Image of dimension $63 \times 63$ where divide aspect vector $=4$ Fig. 2: Image consequences of dimensionality reduction of original image

\section{RGB to HSV (or HSB) color conversion}

The amount of red, green, and blue colors describes RGB color space. Similarly the Hue, Saturation, and Value describes HSV color space.By using RGB color space or HSV color space the color vision can be processed. HSV could be defined by way of many known comaprisons whereas RGB made by arranging basic colors in a group. The "scalex" variable, below in equation (1) [13], signifies the channel scale, for example 255.

$$
\begin{aligned}
& R=\frac{R^{1}}{\text { scale }_{r}}, G=\frac{G^{1}}{\text { scale }_{g}}, B=\frac{B^{1}}{\text { scale }_{b}} \\
& m_{\max }=\max (R, G, B) \\
& m_{\min }=\min (R, G, B) \\
& \Delta=m_{\max }-m_{\min }
\end{aligned}
$$

The hue, $\mathrm{H}$ value is obtained by equation (2) [13] is shown below.

$$
H=\left\{\begin{array}{l}
\text { undefined, } \Delta=0 \\
\frac{G-B}{\Delta}, m_{\max }=R \\
\frac{B-R}{\Delta}+2, m_{\max }=G \\
\frac{R-G}{\Delta}+4, m_{\max }=B
\end{array}\right\}
$$




$$
H^{1}=H \times \text { scale }_{h}
$$

By computing equation (3) [13], the brightness, V is obtained which is based on the bright color channel.

$$
\begin{aligned}
& V=m_{\max } \\
& V^{1}=V \times \text { scale }_{v}
\end{aligned}
$$

The saturation, $\mathrm{S}$, is the variance between the maximum and minimum color channel values, divided by the brightness, $\mathrm{V}$. If $\mathrm{V}$ is 0 , then the resulting saturation is 0 shown in equation (4) [13]

$$
\begin{aligned}
& S=\left\{\begin{array}{l}
0, i f V=0 \\
\frac{\Delta}{V}, \text { otherwise }
\end{array}\right\} \\
& S^{1}=S \times \text { scale }_{s}
\end{aligned}
$$

\section{Experimental Results and Analysis}

The LFW- Labeled Faces in the Wild dataset is a group of JPEG images of well-known persons collected over the internet. Each picture is centered on a single face. The Corel stock photo database with sub-category of 1,000 images named as WANG database. It can retrieve image from a particular category through numerous images similar to retrieval of common stock picture.. The user with query image can search on it to find the relevant image. The proposed CBIR using the partial input image method is tested on Adrien_Brody_0012.jpg image. The Fig.3(a) shows the Original image which the proposed system looking for in the database. The Fig.3(b)-3(f) shows Shaped and Non-shaped Cropped ROI.
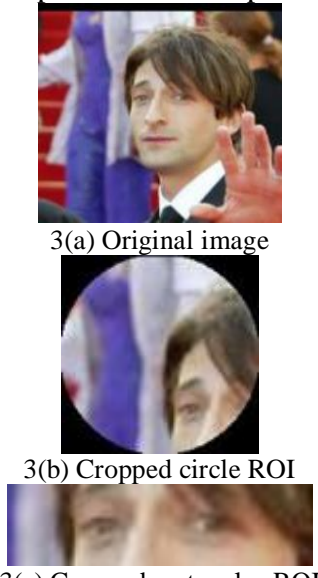

3(c) Cropped rectangles ROI
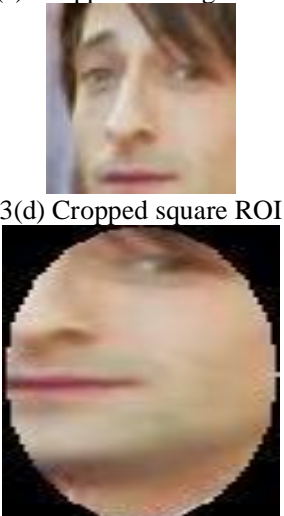

3(e) Cropped elliptical shaped ROI

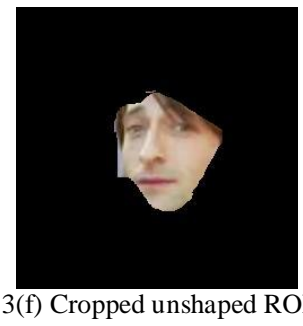

Fig. 3: Original image with shaped and non-shaped cropped images

The outcomes of the proposed algorithm for various shaped and non-shaped ROI are shown in Fig. 4(a)-4(e).
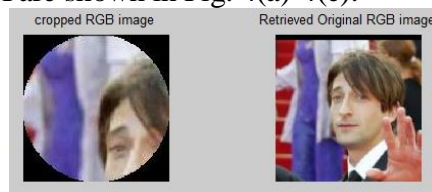

4(a) Output of circle ROI

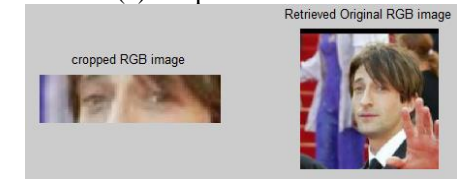

4(b) Output of rectangle ROI

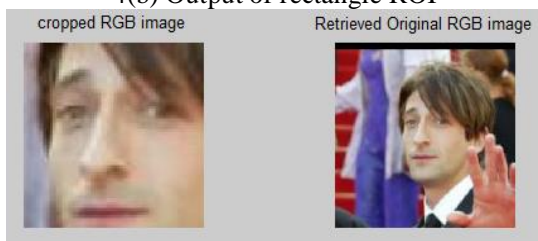

4(c) Output of square ROI

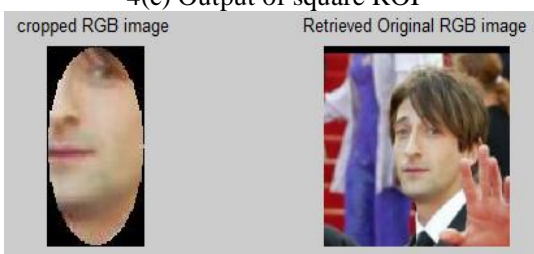

4(d) Output of elliptical shaped ROI

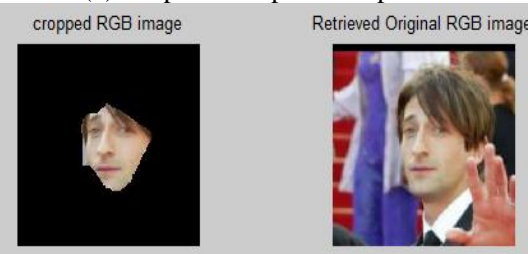

4(e) Output of unshaped ROI

Fig. 4: Image consequences of each step of the proposed method

Table II illustrates the output for the input size of 500 images of various kinds of ROI. The type of ROI, total number of pixels in the cropped image and Elapsed time for retrieval (in seconds) is shown.

Table II: Output for the Input Size of 500 Images for Various Kinds of ROI

\begin{tabular}{|l|l|l|l|}
\hline S.no & Types & $\begin{array}{l}\text { Total number of } \\
\text { pixels in the Cropped } \\
\text { image }\end{array}$ & $\begin{array}{l}\text { Elapsed time for } \\
\text { retrieval (in } \\
\text { seconds) }\end{array}$ \\
\hline 1 & $\begin{array}{l}\text { Cropped Circle } \\
\text { ROI }\end{array}$ & 4096 & 220.14 \\
\hline 2 & $\begin{array}{l}\text { Cropped } \\
\text { Rectangle ROI }\end{array}$ & 3627 & 1191.34 \\
\hline 3 & $\begin{array}{l}\text { Cropped Square } \\
\text { ROI }\end{array}$ & 7569 & 3080.22 \\
\hline 4 & $\begin{array}{l}\text { Cropped } \\
\text { Elliptical shaped } \\
\text { ROI }\end{array}$ & 7560 & 4093.17 \\
\hline 5 & $\begin{array}{l}\text { Cropped } \\
\text { Unshaped ROI }\end{array}$ & 62500 & 4.34 \\
\hline
\end{tabular}


The precision and recall are the assessment metrics normally used in any CBIR system. In this section, the proposed system is tested and the effects are recorded.

The proposed system is tested upon the LFW and WANG databases.

Precision $=\frac{\text { The sum of relevant images retrieved }}{\text { The sum of images retrieved }}$

\section{Recall The sum of relevant images retrieved $=\quad$ The sum of relevant images in the database} The proposed system is tested with 50 images as query image one by one. The performance metrics of the newly designed method results one because when it finds the first matched image, it returns that image as an equivalent of a query image. The proposed system will not consider the replication of the query image.

As per statement in section 3, the matched percentage is fixed to $17 \%$ as the threshold value to find the matched image. The original image is placed in the last position in the database in LFW database. Table III shows output for the data set of various sizes to retrieve Cropped Unshaped ROI. [9]

The same matched percentage is also tested with WANG database. Table IV shows output for the data set of various sizes to retrieve Cropped Unshaped ROI .The original image is placed in the last position in the WANG database.

Table III: Output from LFW Database

\begin{tabular}{|l|l|l|l|}
\hline S.no & $\begin{array}{l}\text { Image set } \\
\text { size }\end{array}$ & $\begin{array}{l}\text { Matched } \\
\text { percentage }\end{array}$ & $\begin{array}{l}\text { Elapsed time (in } \\
\text { seconds) }\end{array}$ \\
\hline 1 & 100 & 38 & 7.72 \\
\hline 2 & 200 & 37 & 9.94 \\
\hline 3 & 300 & 31 & 23.24 \\
\hline 4 & 400 & 34 & 26.53 \\
\hline 5 & 500 & 34 & 24.34 \\
\hline 6 & 1000 & 32 & 46.14 \\
\hline 7 & 1500 & 33 & 127.94 \\
\hline 8 & 2000 & 35 & 199.58 \\
\hline
\end{tabular}

Table IV: Output from Wang Database

\begin{tabular}{|l|l|l|l|}
\hline S.no & $\begin{array}{l}\text { Image set } \\
\text { size }\end{array}$ & $\begin{array}{l}\text { Matched } \\
\text { percentage }\end{array}$ & $\begin{array}{l}\text { Elapsed time (in } \\
\text { seconds) }\end{array}$ \\
\hline 1 & 1000 & 23 & 14.13 \\
\hline 2 & 2000 & 20 & 14.96 \\
\hline 3 & 3000 & 28 & 33.35 \\
\hline 4 & 4000 & 30 & 49.64 \\
\hline 5 & 5000 & 38 & 61.60 \\
\hline 6 & 6000 & 32 & 82.10 \\
\hline 7 & 7000 & 39 & 96.63 \\
\hline 8 & 8000 & 34 & 13.25 \\
\hline 9 & 9000 & 18 & 127.60 \\
\hline 10 & 9990 & 56 & 123.27 \\
\hline
\end{tabular}

Farshad et al. [1] proposed an approach for CBIR using Modified Local Binary Patterns and Morphological Transform. Their approach uses dataset $\mathrm{N}=10,20$ and 40 for measuring performance The Flower set precision is measured distinctly.

The proposed approach achieves $100 \%$ accurate results for $\mathrm{N}$ values ranges from 1000 to 9990 whereas their approach [1] falls below $95 \%$ as shown below. They have done the experiment with a minimum number of samples (10-40 images). But the proposed algorithm is tested with more number of samples (1000-9990 images). Our proposal consistently has shown the better performance than the existing work as shown in Fig.5.[10]

$$
\text { Recognition Accuracy \% Vs Dataset Size }
$$

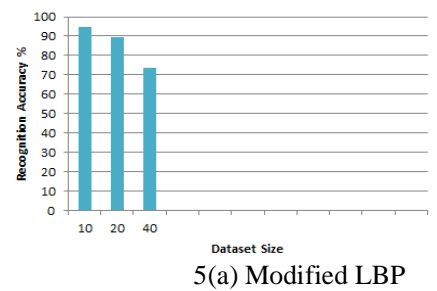

Recognition Accuracy \% Vs Dataset Size

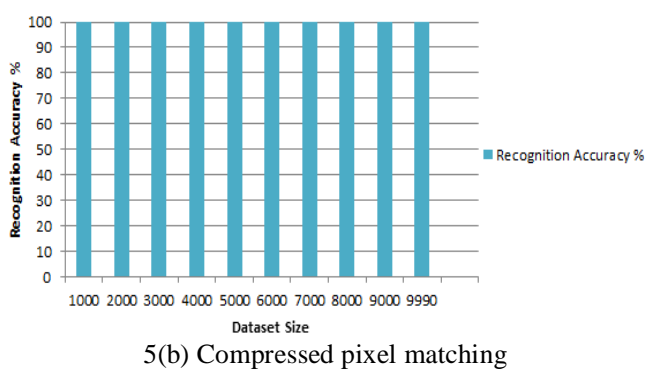

Fig. 5: The comparison of modified LBP with compressed pixel matching algorithm

\section{Conclusion}

In this paper a novel CBIR with partial input image of unshaped images is presented. The working of the newly approached method is assessed using LFW and WANG image database and using the performance metrics, however limited to work on only JPEG images and dataset of considerable size. The outcome of the proposed research helps application like image forensics.

Plentiful fascinating impending research tracks are still there. In addition to addressing present restrictions of proposed system, an interactive system can be created. The image retrieval method with all kinds of images can be created, in which web-scale based can be suggested.

\section{References}

[1] Tajeripour F, Saberi M \& Ershad SF, "Developing a novel approach for content based image retrieval using modified local binary patterns and morphological transform", Int. Arab J. Inf. Technol., Vol.12, No.6,(2015), pp.574-581.

[2] Takahashi T \& Kurita T, "Mixture of subspaces image representation and compact coding for large-scale image retrieval", IEEE transactions on pattern analysis and machine intelligence, Vol.37, No.7,(2015), pp.1469-1479.

[3] Han J \& McKenna SJ, "Query-dependent metric learning for adaptive, content-based image browsing and retrieval”, IET Image Process, Vol.8, (2014), pp.610-618.

[4] Yue J, Li Z, Liu L \& Fu Z, "Content-based image retrieval using color and texture fused features", Mathematical and Computer Modelling, Vol.54, No.3-4,(2011), pp.1121-1127.

[5] Elleuch Z \& Marzouki K, "Multi-index structure based on SIFT and color features for large scale image retrieval", Multimed Tools Appl, (2016).

[6] Khokher A \& Talwar R, "A fast and effective image retrieval scheme using color, texture, and shape-based histograms", Multimed Tools Appl, (2016).

[7] Deepika, J., Senthil, T., Rajan, C., \& Surendar, A. (2018). Machine learning algorithms : a background artifact. International Journal of Engineering \& Technology, 7, 143-149

[8] Xu B, Bu J, Chen C, Wang C, Cai D \& He X, "EMR: A scalable graph-based ranking model for content-based image retrieval", IEEE Transactions on knowledge and data engineering, Vol.27, No.1,(2015), pp.102-114.

[9] G Mussabekova, S Chakanova, A Boranbayeva, A Utebayeva, K Kazybaeva, K Alshynbaev (2018). Structural conceptual model of forming readiness for innovative activity of future teachers in general education school. Opción, Año 33. 217-240

[10] G Cely Galindo (2017) Del Prometeo griego al de la era-biós de la tecnociencia. Reflexiones bioéticas Opción, Año 33, No. 82 (2017):114-133 